\title{
Situación actual de la investigación etnobotánica sobre palmeras de Perú
}

\author{
Current status of ethnobotany research on palms from Peru
}

Joaquina Albán', Betty Millán' y Francis Kahn²

1 Museo de Historia Natural, Universidad Nacional Mayor de San Marcos, Lima - Perú. Avda. Arenales 1256 Jesús María, Lima 14, Perú. Email Betty Millán: bmillans@unmsm.edu.pe,bmillans@ gmail.com

2IRD, UMR188-DIAPC, DYNADIV, Casilla 18-1209, Lima, Perú

Trabajo presentado al Simposio Internacional "LAS PALMERAS EN EL MARCO DE LA INVESTIGACIÓN PARA EL DESARROLLO EN AMÉRICA DEL SUR", del 07 al 09 de Noviembre 2007, Museo de Historia Natural, Universidad Nacional Mayor de San Marcos, Lima, Perú.

Publicado online: 29/11/2008

\section{Resumen}

Se evalúa la situación actual de la investigación en etnobotánica y botánica económica de las palmeras peruanas considerando el conocimiento relativo a los usos y a los nombres vernáculos, y el estado de la producción científica en esas áreas. Por lo menos un uso fue registrado en 104 de las 136 especies peruanas. Fueron registrados un total de 268 diferentes usos en 16 categorías. Los usos más frecuentes fueron de las categorías "construcción", "comestible", "artesanal” y "medicinal”. Son 109 las especies que tienen nombres vernáculos en el Perú. La producción científica fue analizada considerando 4 categorías: (i) estudios de botánica económica general, (ii) estudios etnográficos y de etnobiología, (iii) estudios en palmeras americanas de interés económico, (iv) estudios que tratan exclusivamente de las palmeras peruanas útiles. Se concluye que el conocimiento en etnobotánica y botánica económica de las palmeras peruanas es esencialmente descriptivo, con numerosas informaciones repetidas. Muy pocos son los trabajos que se dedican a las especies de interés económico con la finalidad de producir un progreso efectivo en el conocimiento de las especies y que conlleven a un mejoramiento genético o agronómico.

Palabras clave: Arecaceae, etnobotánica, botánica económica, Perú

\section{Abstract}

The current situation concerning research in ethnobotany and economic botany of Peruvian palms is analyzed through a review of the literature with emphasis on knowledge related to uses and vernacular names. Of the 136 Peruvian palm species, 104 have at least one use. A total of 268 different uses distributed in 16 categories were registered. The most frequent categories are "construction", "edible", "craft industry" and "medicinal". There are 109 palm species with at least one vernacular name in Peru. The consulted literature is analyzed in four categories: (i) general studies in economic botany, (ii) ethnographic and ethnobiological studies, (iii) studies of South-American palms of economic interest, (iv) studies that exclusively deal with the useful Peruvian palms. Ethnobotanical knowledge of Peruvian palms proves to be essentially descriptive, with much repetitive information. Studies that significantly contribute to the genetic or agronomical improvement of the economically promising palms are rare.

Keywords: Arecaceae, ethnobotanics, economic botany, Peru

\section{Introducción}

En el presente trabajo se revisa el conjunto de los trabajos hechos en palmeras peruanas sobre etnobotánica y botánica económica. Los trabajos sobre palmeras presentan un enfoque taxonómico o ecológico y proveen informaciones etnobotánicas; otros tratan de la importancia económica de las palmas nativas y de su posible implicancia dentro de un marco de desarrollo sostenible, ya sea por el manejo organizado de las poblaciones naturales, por el desarrollo de sistemas agroforestales, o por la recuperación de los suelos degradados y de los espacios deforestados.

En primer lugar analizamos la importancia de las especies en relación a las diferentes categorías de usos. Se consideran luego las publicaciones que consideran a las palmeras peruanas útiles. El cruzamiento de los dos procedimientos nos permite apreciar las áreas de mayor conocimiento y las que todavía carecen de contribución y necesitan mayor esfuerzo de investigación.

Con los resultados obtenidos sugerimos algunas líneas que deberían seguirse para que las investigaciones en etnobotánica y la botánica económica de las palmeras peruanas respondan a los grandes retos de nuestro tiempo.

\section{Material y métodos}

Lista de las especies peruanas - Los cambios constantes de interpretación en el área de la taxonomía exigen referirse a la última lista de especies consideradas validas y publicadas por especialistas reconocidos. Seguiremos aquí la lista producida por Govaerts y Dransfield (2005) modificada conforme a las interpretaciones, presentada en el presente volumen, sobre las palmeras de América del Sur (Pintaud et al., 2008).

Datos sobre los usos y nombres vernáculos - Se revisó el mayor número de publicaciones con informacion directa o indirecta sobre la etnobotánica de las palmeras peruanas. Se registraron las informaciones de los colectores de plantas en los Herbarios de Lima, Loreto, y La Libertad. También se incluyen datos obtenidos en encuestas realizadas por J. Albán y B. Millán como contribución en varios proyectos etnobotánicos que se han realizado en los últimos 25 años en las comunidades nativas siguientes: Amahuaca (río Bocapariamanu, Madre de Dios), Nahuas (río Serjali, Ucayali), Shipibo Conibo (río Ucayali, Ucayali), Ashaninca (San Pedro de Pichanaz, Junin), Aguaruna (río Marañon, Amazonas), Huambisa (río Santiago, Loreto), Cocama-Cocamilla (río Pacaya-Samiria, Loreto).

En el presente estudio, se adopta la clasificación de usos propuesta por Albán (1985), que considera 16 categorías (Tabla 1).

Solamente los nombres y usos más comunes a nivel regional fueron considerados. No se listaron los nombres en idiomas nativos, porque además de no tener disponible los inventarios para todas las etnias presentes en el país, aquellos nombres en idiomas nativos, a menudo, carecen de las referencias de herbario para comprobar la identificación de la especie.

Fuentes bibliográficas — La información sobre las palmeras peruanas se encuentra dispersa en numerosos trabajos publicados que cubren una gran gama de temas referentes a las plantas 
Tabla 1. Frecuencia de registros por categoría de uso. ${ }^{1}$ Techado, horcones, pisos, puertas, muebles, postes calles. ${ }^{2}$ Fruto, palmito - de consumo intensivo u ocasional. ${ }^{3}$ Utensilios, artículos de caza, pesca, souvenirs, decoración personal, soguillas para amarres de canastas, corchos para botellas, etc. ${ }^{4}$ Ritual mágico, religioso. ${ }^{5}$ Rhynchophorus palmarum (Coleoptera, Curculionidae).

\begin{tabular}{lc} 
Construcción $^{1}$ & 66 \\
Alimenticio $^{2}$ & 61 \\
Artesanal $^{3}$ & 59 \\
Medicinal & 29 \\
Ceremonial $^{4}$ & 8 \\
Cosméticos & 6 \\
Extracción de sal vegetal & 6 \\
Aceite & 5 \\
Ornamental & 5 \\
Carnada para peces & 4 \\
Cultivo de curculiónido ${ }^{5}$ (suri") & 4 \\
Decorativo & 4 \\
Alimento para animales & 3 \\
Fibra & 3 \\
Perfumería & 3 \\
Tintóreo & 3 \\
Total registros & 268 \\
\hline
\end{tabular}

útiles en general, o a las palmeras por región o por país. Se trata de sistemática, ecología, usos, farmacopeas, listas de nombres vernáculos, así como de economía a nivel local y regional, potencial para la industria alimentaria u otra. También proveen informaciones validas los trabajos que analizan sistemas tradicionales de manejo forestal, extractivista, agroforestal y huertos.

Se han repartido los trabajos en cuatro categorías: (i) estudios de etnobotánica, botánica económica en general; (ii) estudios de etnografía y etnobiología realizados por antropólogos; (iii) estudios de etnobotánica y botánica económica de palmeras incluyendo especies peruanas, y estudios taxonómicos de palmeras en los que se citan usos y nombres vernáculos de especies peruanas; (iv) estudios realizados en el Perú que tratan exclusivamente de palmeras nativas, o que incluyen análisis avanzados sobre poblaciones peruanas de palmeras en un marco más amplio.

\section{Resultados}

Uso de las palmeras en el Perú (Tablas 1 y 2) - Se ha registrado al menos un uso en 104 (76,5\%) de las 136 especies peruanas; 32 especies no tienen usos conocidos en el país. Sin embargo, se han reportado usos en otros países para algunas de estas últimas (p.e. Astrocaryum huaimi, Martius 1844).

Se han registrado 268 usos: 33 especies con sólo un uso, 25 con dos usos, y 46 especies con 3 o más usos (hasta 9). Los más frecuentes son de las categorías "construcción”, "alimenticio", "artesanal" y "medicinal” con 66, 61, 59 y 29 registros respectivamente.

Nombres vernáculos (Tabla 2) - Los registros de nombres vernáculos muestran una situación similar a la de los usos: 109 especies $(80,1 \%)$ de palmeras peruanas tiene uno o más nombres vernáculos de uso compartido, es decir no específico a una etnía particular.

\section{Análisis de la literatura en etnobotánica y botánica económica de palmeras}

(1) Estudios de etnobotánica y botánica económica en general. Son numerosos los trabajos que responden a estos criterios. Se consideran aquí dos tipos de trabajos:

a. las publicaciones de connotación regional que se refieren a palmeras que también se encuentran en el Perú (p.e. Balick, 1985; Cavalcante, 1974; Goulding y Smith, 2007; Grenand et al., 2004; Pesce, 1985; Peters et al., 1989a,b; Schultes y Rauffauf, 1990);

b. los trabajos realizados en el Perú que se refieren, entre otras plantas, a palmeras peruanas (Albán, 1985, 1994; Aldave y Mostacero, 1988: Arellano, 1992; Arévalo, 1994; Baluarte, 2000; Barriga, 1994; Bodmer et al., 1999; Brack, 1999; Campos y Galán, 1989; Cavero, 1941; Chang y Aymara, 1987; Dañobeytia, 2002; Didir, 1995; Flores Paitan, 1998; Gari, 2001; La Torre-Cuadros, 1998; Mejía y Rengifo, 1995; Padoch y Jong, 1990; Pérez, 2002; Peters et Hammond, 1990; Pennington et al., 2004; Phillips, 1993; Piñedo-Vásquez et al., 1990, 1992; Rengifo y Cerruti, 1997; Salo y Torres Vásquez, 1998; Soukup, 1962, 1963, 1964; Stagegaard et al., 2002; Ugent y Ochoa, 2006; Vásquez, 1999; Vásquez y Gentry, 1989; Yacovleff y Herrera, 1935).

(2) Estudios etnográficos y de etnobiología realizados por antropólogos destacando las nomenclaturas y los sistemas de clasificación de las plantas por los nativos del Perú, y que consideran las palmeras (Alexiades, 1999; Berlin, 1970, 1977, 1992; Berlin y Berlin, 1979a,b; Bodley, 1978; Bodley y Benson, 1979), o de países vecinos (p.e. Alvarado, 1956; Descola, 1986, 1987, 1993; Lévi-Strauss, 1968; Tournon, 2002; Tournon et al., 1986; Yákuam’jintia y Péaskantáshishtik, 1991).

(3) Estudios de etnobotánica y botánica económica de palmeras incluyendo especies peruanas, y estudios taxonómicos de palmeras en los que se citan usos y nombres vernáculos de especies peruanas.

Entre los trabajos a nivel regional se pueden citar los de Balick (1984, 1989), Balick y Beck (1990), Balslev y Moraes, (1989), Bernal (1992), Borchsenius y Moraes (2006), Borchsenius et al. (1998), Borgoft Pedersen y Balslev (1990), Byg y Balslev (2004), Clement (1992), Dugand (1972), Gómez et al. (1996), Henderson (1995), Henderson et al. (1995), Johnson (1986), Kahn (1993, 1997), Kahn y Granville (1991, 1992), Kahn y Moussa (1995), Lleras (1985), Lleras y Coradin (1988), Moussa y Kahn (1997), Moussa et al. (1998), Paniagua Zambrana (2005), Paniagua Zambrana et al. (2007), Schultes (1977), Wallace (1853).

Esta categoría también incluye trabajos mas específicos, por ejemplo con las especies siguientes: Aphandra natalia (Borgtoft Pedersen, 1992), Astrocaryum chambira (Holm Jensen y Balslev, 1995; Wheeler, 1970), Attalea maripa (Blaak, 1983), Bactris gasipaes (Clement, 1988; Clement y Mora Urpi, 1988; Couvreur et al., 2007), Euterpe precatoria (Castro, 1993; Rocha, 2004), Oenocarpus bataua (Balick, 1986, 1988; Balick y Anderson, 1986/1987; Balick y Gershoff, 1981; Pinto 1951), Mauritia flexuosa (Altman y Cordeiro, 1964; González Boscán, 1978; Mariath et al., 1989; Ramírez y Brito, 1990; Urrego Giraldo, 1987). 
Tabla 2. Las especies de palmeras del Perú con los nombres vernáculos más comunes y las categorías de usos principales. s.n.: sin nombre vernáculo; n.r.: uso no registrado.

1. Aiphanes deltoidea - shicashica - n.r.

2. Aiphanes eggersii - s.n. - alimenticio

3. Aiphanes horrida - s.n. - n.r.

4. Aiphanes spicata - s.n. - n.r.

5. Aiphanes ulei - chontilla - n.r.

6. Aiphanes weberbaueri - chontilla - n.r.

7. Aphandra natalia - piasaba - alimenticio, artesanal, construcción, fibra

8. Astrocaryum carnosum - huicungo - alimenticio

9. Astrocaryum chambira - chambira - alimenticio, artesanal, cosmético, fibra, medicinal

10. Astrocaryum chonta - huicungo - alimenticio, artesanal, medicinal

11. Astrocaryum faranae - huicungo - alimenticio

12. Astrocaryum gratum - huicungo - alimenticio

13. Astrocaryum gynacanthum - s.n. - n.r.

14. Astrocaryum huaimi - s.n. - n.r.

15. Astrocaryum huicungo - huicungo - alimenticio

16. Astrocaryum jauari - chambirilla, huiririma, - alimenticio, artesanal, carnada para pesca, construcción, medicinal

17. Astrocaryum javarense - huicungo - alimenticio, artesanal

18. Astrocaryum macrocalyx - huicungo - alimenticio, artesanal

19. Astrocaryum perangustatum - coquito-del-monte, huicungo - alimenticio

20. Astrocaryum scopatum - huicungo - alimenticio, artesanal

21. Astrocaryum urostachys - huicungo - alimenticio

22. Attalea bassleriana - shapaja, shebon - alimenticio, artesanal, construcción, "suri"

23. Attalea butyracea - shapaja, shebon - alimenticio, artesanal, ceremonial, construcción, medicinal, "suri"

24. Attalea cephalotus - shebon - construcción

25. Attalea ferruginea - coquito - alimenticio, artesanal, construcción

26. Attalea insignis - contillo, shebon - alimenticio, artesanal, construcción, medicinal,

27. Attalea kewensis - s.n. - n.r.

28. Attalea maripa - cucurito, inayuga, wacha - alimenticio, artesanal, construcción, "suri"

29. Attalea moorei - shebon - construcción

30. Attalea peruviana - s.n. - n.r.

31. Attalea phalerata - shapaja, shebon - alimenticio, artesanal, aceite, ceremonial, construcción, cosmético, decorativo, medicinal, sal vegetal

32. Attalea plowmanii - shapajilla - alimenticio, construcción

33. Attalea polysticha - catirina - construcción

34. Attalea salazarii - s.n. - n.r.

35. Attalea tessmannii - conta - alimenticio, artesanal, medicinal, carnada para pesca

36. Attalea weberbaueri - s.n. - n.r.

37. Bactris acanthocarpa - chontilla, ñeja, ñejilla - alimenticio, construcción, medicinal

38. Bactris bidentula - ñejilla - alimenticio, artesanal

39. Bactris bifida - ñeja-negra - alimenticio

40. Bactris brongniartii - ñejilla - alimenticio, artesanal

41. Bactris chaveziae - s.n. - n.r.

42. Bactris coloniata - s.n. - n.r.

43. Bactris concinna - ñejilla, ñejilla-de-altura - alimenticio, artesanal, construcción

44. Bactris corossilla - chontilla, ñejilla - alimenticio, construcción

45. Bactris fissifrons - nejilla - n.r.

46. Bactris gasipaes - pijuayo - (cultivada), alimenticio, artesanal, construcción, cosmético, medicinal, tintóreo

47. Bactris halmoorei - ñejllla - alimenticio, artesanal, construcción

48. Bactris hirta - ñejilla - alimenticio

49. Bactris killipii - ñejilla - n.r.

50. Bactris macroacantha - ñejilla - alimenticio

51. Bactris major - cubarro - artesanal, construcción

52. Bactris maraja - ñejilla, chontilla, chambira-ñeja, dinamillo-de-altura - alimenticio, artesanal

53. Bactris martiana - s.n. - alimenticio

54. Bactris riparia - chontilla, ñejilla - alimenticio, medicinal

55. Bactris schultesii - ñejilla - alimenticio, construcción

56. Bactris setulosa - chonta - n.r.

57. Bactris simplicifrons - chontilla, ñejilla - artesanal, construcción, medicinal

58. Bactris sphaerocarpa - ñejilla - n.r.

59. Ceroxylon echinulatum - palma-del-ramo, palma-real - alimenticio, construcción, ceremonial

60. Ceroxylon parvifrons - palma-real - ceremonial, construcción

61. Ceroxylon paroum - palma-del-tamban - construcción

(Continúa...) 


\section{Tabla 2. Continuación...}

62. Ceroxylon quindiuense - s.n. - n.r.

63. Ceroxylon vogelianum - coco - alimenticio, ceremonial, construcción

64. Ceroxylon weberbaueri - s.n. - construcción

65. Chamaedorea angustisecta - sangapilla - n.r.

66. Chamaedorea fragrans - sangapilla, shicashica - perfumería

67. Chamaedorea linearis - caña-agria, chontilla, coroso, palmito - ornamental

68. Chamaedorea pauciflora - desodorante-indígena, ponilla, sangapilla - perfumería

69. Chamaedorea pinnatifrons - chontilla, cashaponilla, cashipana - alimenticio, artesanal, construcción

70. Chelyocarpus repens - sacha-bombonaje - alimenticio, artesanal, construcción

71. Chelyocarpus ulei - sacha-aguajillo, sacha-bombonaje - alimenticio, artesanal, sal vegetal, ornamental

72. Desmoncus giganteus - chontilla-del-diablo, varacasha - artesanal, medicinal

73. Desmoncus mitis - varacasha - artesanal, ceremonial

74. Desmoncus orthacanthos - pijuajillo, varacasha - artesanal, medicinal

75. Desmoncus polyacanthos - cashavara, pijuajillo, varacasha - artesanal

76. Dictyocaryum lamarckianum - basanco - construcción

77. Dictyocaryum ptarianum - pona-colorada - construcción

78. Elaeis oleifera - poloponta - alimenticio, aceite, construcción, medicinal

79. Euterpe catinga - chonta, huasai - alimenticio, artesanal, medicinal

80. Euterpe luminosa - palma-palanca, guayaquil - construcción

81. Euterpe precatoria - huasai - alimenticio, artesanal, ceremonial, construcción, decorativo, medicinal, sal vegetal,

82. Geonoma atrovirens - calzón-panga - alimenticio, artesanal, construcción

83. Geonoma brongniartii - aliman, culluli, palmiche - construcción, perfumería

84. Geonoma camana - chontilla, palmiche - construcción, sal vegetal

85. Geonoma deversa - barobaro, chontilla, palmiche, pulune - artesanal, construcción, sal vegetal

86. Geonoma euspatha - s.n. - n.r.

87. Geonoma interrupta - chontilla - artesanal, construcción

88. Geonoma laxiflora - ponilla - artesanal

89. Geonoma leptospadix - palmito, sangapilla-masha - artesanal, construcción

90. Geonoma longepedunculata - palmiche - construcción

91. Geonoma macrostachys - calzón-panguillo, palmichillo, palmiche, ponilla - artesanal, construcción, ornamental

92. Geonoma maxima - palmiche, palmiche-negro - artesanal, construcción, medicinal

93. Geonoma megalospatha - s.n. - n.r.

94. Geonoma occidentalis - s.n. - n.r.

95. Geonoma orbignyana - chile - artesanal

96. Geonoma poeppigiana - palmiche-colorado, palmichillo - construcción

97. Geonoma pycnostachys - palmiche - n.r.

98. Geonoma supracostata - s.n. - n.r.

99. Geonoma triglochin - gaban - artesanal, construcción,

100. Geonoma trigona - s.n. - n.r.

101. Geonoma undata - caña-brava, chontilla, micana - artesanal, construcción, ornamental, tintóreo

102. Hyospathe elegans - palma-de-tinta, palmichillo, palmicho - artesanal, construcción, medicinal

103. Hyospathe macrorhachis - s.n. - n.r.

104. Hyospathe peruviana - s.n. - n.r.

105. Iriartea deltoidea - huacrapona - alimento animales, artesanal, ceremonial, construcción, cosmético, decorativo, medicinal

106. Iriartella stenocarpa - cashaponita - artesanal, construcción, medicinal

107. Itaya amicorum - sacha-bombonaje - construcción, sal vegetal

108. Lepidocaryum tenue - irapay - artesanal, construcción, medicinal

109. Manicaria saccifera - busu - alimenticio, artesanal, construcción, medicinal

110. Mauritia carana - aguaje-de-varillal, canangucha - alimenticio, artesanal, construcción

111. Mauritia flexuosa - aguaje - alimenticio, alimento animales, artesanal, construcción, decorativo, fibra, medicinal, "suri"

112. Mauritiella aculeata - aguajillo, cananguchillo - alimenticio, artesanal, construcción

113. Mauritiella armata - aguarillo - alimenticio, artesanal, construcción

114. Oenocarpus balickii - sinamillo - alimenticio, aceite

115. Oenocarpus bataua - sacumana, ungurahui - alimenticio, aceite, artesanal, construcción, cosmético, medicinal

116. Oenocarpus mapora - ciamba, sinamillo - alimenticio, aceite, artesanal, construcción, medicinal

117. Pholidostachys synanthera - campana, palmiche grande - alimenticio, construcción

118. Phytelephas macrocarpa - yarina - alimenticio, artesanal, construcción, medicinal

119. Phytelephas tenuicaulis - tagua, yarina - alimenticio, artesanal, construcción

120. Prestoea acuminata - palmo, tinguiso - alimenticio, carnada para pesca

121. Prestoea carderi - s.n. - n.r.

122. Prestoea ensiformis - caño, cuyol, palmiche, palmita - alimenticio

(Continúa...) 


\section{Tabla 2. Continuación...}

123. Prestoea schultzeana - chincha, chincha-yura - alimenticio, carnada para pesca, construcción, medicinal

124. Socratea exorrhiza - cashapona - alimento animales, artesanal, construcción, medicinal, cosmético

125. Socratea rostrata - s.n. - n.r.

126. Socratea salazarii - cashapona-de-altura - artesanal

127. Syagrus sancona - cuya, sappan-de-venado - artesanal

128. Syagrus smithii - s.n. - alimenticio, construcción

129. Welfia regia - camara, palma-amarga - construcción

130. Wendlandiella gracilis - palmerilla, ponilla, shontilla - ornamental

131. Wettinia aequatorialis - s.n. - n.r.

132. Wettinia augusta - ponilla, ponilla, sachapona - artesanal, construcción, medicinal

133. Wettinia drudei - ponilla - construcción

134. Wettinia longipetala - s.n. - n.r.

135. Wettinia maynensis - ponilla, pullo-coroto - alimenticio, artesanal, construcción

136. Wettinia minima - s.n. - n.r.

(4) Estudios realizados en el Perú que tratan exclusivamente de palmeras nativas, o que incluyen análisis avanzados sobre poblaciones peruanas de palmeras en un marco más amplio. Se incluyen en esta categoría:

a. estudios sobre palmeras peruanas dando informaciones sobre los usos (Balslev et al., 2008; Gutierrez, 1987; Johnson, 1975; Kahn y Mejía, 1988; Kahn y Moussa, 1994; López Parodi, 1988; Mejía, 1983, 1988; Millán, 1998; Moore et al., 1960; Van der Linden y López, 1990; Vormisto, 2002a), o analizando sus clasificaciones por los nativos (Gullart, 1968; Roca, 2004 en los Aguajuna y Huambisa).

b. estudios sobre la importancia de productos de palmeras en los circuitos comerciales y mercados - Mejía (1992), Padoch (1988), Vormisto (2002b).

c. estudios sobre la ecología, estructura de población, manejo de poblaciones - Boll et al. (2005), Castagne (1971), González Rivadeneyra (1971), Kahn (1988, 1990, 1991), Ruiz Murrieta (1993).

d. estudios sobre la germinación de especies promisorias López 1968, López Mori (1984), Ramírez (1974), Rojas Ruiz (1985).

e. estudios de caracterización morfológica - Rojas Ruiz (1992)

f. estudios de caracterización molecular - Barcelos et al. (1999, Elaeis oleifera); Montufar (2007, Oenocarpus bataua). En ambos se analizan poblaciones peruanas con otras de diferentes países.

g. estudios sobre el potencial de las especies nativas en la industria conservera de palmito, de pulpa, la industria papelera - Alva Arévalo (1988), Araujo Abanto (1982), Bresani (1924), De Los Heros et al. (1980/1981), Salazar y Roessl (1977).

h. estudios del potencial de las palmeras nativas para producción de aceite, análisis del valor nutricional, vitaminas — Jordán Ledesma (1986), Lognay et al. (1987), Schwyzer (1978), Testua Vásquez y Aspejo Dávila (1987), Trebejos et al. (1991). Campos Rivera (1988) analiza el aceite extraído de los gusanos que crecen en los troncos de varias especies de palmeras.
Son 36 los trabajos que se consagran a una sola especie; entre estos, 24 se dedican a Mauritia flexuosa:

Aphandra natalia (1) — Boll et al. (2005).

Astrocaryum chambira (2) — Rojas Ruiz (1992), Vormisto (2002b).

Desmoncus, D. polyacanthos (2) - Henderson y Chávez (1993), Hübschmann et al. (2007).

Elaeis oleifera (2) - Barcelos et al. (1999), Kahn y Mejía (1986).

Iriartea deltoidea (1) — Johnson y Mejía (1998).

Lepidocaryum tenue (1) — Kahn y Mejía (1987).

Mauritia flexuosa (24) - Almanza (1967), Alva Arévalo (1988), Araujo Abanto (1982), Bohórquez (1976), Bresani (1924), Castagne (1971), De Los Heros y Zárate (1980/1981), Del Castillo et al. (2006), González Rivadeneyra (1971), Jordán Ledesma (1986), Kahn et al. (1993), Lognay et al. (1987), López (1968), López Mori (1984), Noriega Monteiro (1980), Padoch (1988), Paramonga (1965), Ramírez (1974), Ruiz Murrieta (1991, 1993), Ruiz Murrieta y Levistre (1993), Salazar (1967), Salazar y Roessl (1977), Testua Vásquez y Aspejo Dávila (1987).

Oenocarpus bataua (3) - Montufar (2007), Schwyzer (1978), Trebejos et al. (1991).

\section{Conclusiones}

La compilación de datos sobre los usos muestra que, con más del 70\% de especies utilizadas por el hombre, las palmeras constituyen el recurso vegetal con mayor utilidad registrado entre los pobladores de la selva peruana. A pesar de la compilación, no se puede excluir la posibilidad de usos para otras especies. Sin embargo, podemos asumir que se trata de usos secundarios y ocasionales y que no son registrados en obras especializadas o sintéticas de mayor difusión.

Gran número de informaciones sobre las palmeras útiles del Perú se encuentra en obras que no enfocan sólo a especies peruanas. Los datos registrados son bastante repetitivos, siendo recuperados de un trabajo al otro. Las fuentes originales de conocimiento de usos se deben buscar en los trabajos de caracteres etnográficos, o en los estudios etnobotánicos consagrados a comunidades étnicas particulares o a una región. 
En ocho especies, se registraron trabajos sólo dedicados a ellas, siendo claramente Mauritia flexuosa, la especie estudiada con mayor frecuencia. Sin embargo, varios de estos estudios se limitan a informes preliminares o tesis de difusión muy limitada. Para algunas especies importantes en la economía regional hay escasez de referencias. Es el caso de Bactris gasipaes. Material no publicado de difusión interna se puede encontrar en instituciones como el Instituto Nacional de Innovación y Extensión Agraria (INIA).

En realidad, pocos son los trabajos que se dedican a las especies de interés económico con perspectiva de contribuir a su mejoramiento genético o agronómico y que marquen un progreso efectivo en el conocimiento de esas especies.

\section{Perspectivas para las investigaciones etnobotáni- cas en el Perú}

Es necesario proseguir la recolección de datos en las diversas comunidades étnicas por tratarse de un patrimonio cultural de la humanidad, además de ser un conocimiento en peligro de extinción.

Sin embargo, la relativa redundancia de los inventarios de usos de las palmeras realizados en los pueblos selváticos da prueba del buen nivel de los conocimientos adquiridos. Aparece también claramente que estos conocimientos no se traducen a nivel del desarrollo para una valorización de esta biodiversidad.

Hay que reconocer que el entusiasmo con el cual la comunidad científica destacaba el potencial económico de las palmeras nativas en los ańos 80 y 90 contrasta con el impacto casi nulo de resultados en términos de desarrollo. Todos estaban de acuerdo que tanto el conocimiento de la biología y ecología de las palmeras promisorias como la valoración de sus productos se imponían como una de las prioridades para acompañar el proceso de domesticación. Sin embargo, muy poco se ha hecho en este sentido.

La situación se puede entender. La agroindustria se ha desarrollado mucho con solamente tres palmeras cultivadas, el cocotero, la palmera aceitera africana y la palmera datilera, que monopolizan el esfuerzo de innovación. Sus productos y derivados alimentan diariamente los circuitos comerciales a nivel mundial. Casi no hay espacio para las palmeras nativas, muy atrasadas en el proceso de mejoramiento genético y agronómico. ¿Cuál podría ser entonces la fuerza de los argumentos para convencer los proveedores de fondos de invertir en la investigación sobre las palmeras nativas con resultados a largo plazo cuando la inversión en las tres palmeras cultivadas es obviamente mucho más rentable a corto plazo?

La etnobotánica no puede quedarse en los inventarios, tampoco puede limitarse a la producción de libros y artículos en revistas científicas. Las investigaciones en etnobotánica y botánica económica deben responder a las exigencias de un mundo que tiene, para asegurar su futuro, que aprovechar su biodiversidad en el marco de un desarrollo sostenible.

En este contexto, es necesario que las investigaciones etnobotánicas pasen a ser más activas, contribuyendo a encontrar soluciones para manejar con mayor eficiencia los recursos representados por dichas palmeras, no sólo al analizar los sistemas de explotación sino también al contribuir en innovaciones para transformar los productos y facilitar su integración en circuitos comerciales de mayor importancia. Si el botánico o el etnobotánico no se encuentra en mejores condiciones para desarrollar esas innovaciones, tiene sin embargo la posibilidad de asociar a sus proyectos ingenieros y empresarios que tienen las competencias para ello.

Las investigaciones deben escapar del mundo académico; es un punto inevitable para que la etnobotánica se adapte a la realidad del mundo actual. Tomarán su sentido en ser participativas con la implicación efectiva de las comunidades directamente interesadas en los resultados. Los habitantes del mundo rural quiénes siempre han proporcionado la información a los investigadores se convertirán en protagonistas de la investigación y serán directamente implicados y responsables tanto en la definición de los objetivos, en la obtención y la elaboración de los resultados como en su difusión y valorización.

\section{Literatura citada}

Albán J.A. 1985 Un registro de datos etnobotánicos. Boletín de Lima, (7) 39: 93-96.

Albán J.A. 1994. La mujer y las plantas útiles silvestres en la comunidad Cocama-Cocamilla de los ríos Samiria y Marañon. Informe. Proyecto WWW 7560. Lima, 85 p.

Aldave A. \& J. Mostacero. 1988. Botánica farmacéutica. Editorial Libertad, Lima.

Alexiades M.N. 1999. Ethnobotany of the Ese eja: plants, health and change in an Amazonian society. PhD dissertation, The City University of New York.

Almanza O.B. 1967. El aguaje: milagro de los pantanos selváticos. Industría Peruana, 145: 40-41.

Altman R.R.A. \& M.M.C.M. Cordeiro. 1964. A industrialização do fruto do buriti (Mauritia vinifera Mart. ou M. flexuosa). INPA Publ. 5 Química, Manaus.

Alva Arévalo A.G. 1988. Elaboración y conservación por calor de pulpa y néctar a partir del fruto de aguaje (Mauritia flexuosa). Tesis Bach. UNAP, Iquitos.

Alvarado L. 1956. Datos etnográficos de Venezuela. Ed. Rangon C.A., Caracas.

Araujo Abanto V.A. 1982. Pulpa química y semiquímica al sulfato de hojas de palma aceitera (Elaeis guineensis Jacq.) y de pecíolos de aguaje (Mauritia flexuosa L.f.). Tesis Ing. forestal, UNALM, Lima.

Arellano P. 1992. El Libro Verde. Guía de recursos terapéuticos vegetales I. INMETRA/MINSA, Lima.

Arévalo G. 1994. Las plantas medicinales y sus beneficios en la salud Shipibo-conibo. AIDESEP. Ucayali.

Balick M.J. 1984. Ethnobotany of palms in the neotropics. Advances in Economic Botany 1: 9-23.

Balick M.J. 1985. Useful plants of Amazonia: A resource of global importance. In: Prance G.T., Lovejoy, T.E. (eds). Key environments: Amazônia. Pergamon Press, Oxford, New York, pp.339-368.

Balick M.J. 1986. Systematics and economic botany of the Oenocarpus-Jessenia (Palmae) complex. Advances in Economic Botany, 3: 1-140.

Balick M.J. 1988. Jessenia and Oenocarpus: neotropical oil palms worthy of domestication. FAO plant production and protection paper 88 , Roma.

Balick M.J. 1989. Native neotropical palms: a resource of global interest. In: Wickens, G.E., Haq, N. \& P. Day. New crops for food and industry, Chapman and Hall, London, pp. 323-332.

Balick M.J. \& A.B. Anderson. 1986/1987. Dry matter allocation in Jessenia bataua (Palmae). Acta Amazonica, 16/17: 135-139. 
Balick M.J. \& H.T. Beck. 1991. Useful palms of the world. A synoptic bibliography. Columbia University Press, New York.

Balick M.J. \& S.N. Gershoff. 1981. Nutritional evaluation of the Jessenia bataua palm: source of high quality protein and oil for Tropical América. Economic Botany, 35: 261-271.

Balslev H. \& M. Moraes. 1989 - Sinopsis de las palmeras de Bolivia. AAU Reports 20, Aarhus.

Balslev H., C. Grandez, N.Y. Paniagua Zambrana, A.L. Moller \& S.L. Hansen. 2008. Palmas (Arecaceae) útiles en los alrededores de Iquitos, Amazonia Peruana. Rev. per. biol. 15(supl.1): 121-132

Baluarte J. 2000. La manufactura de muebles a partir de productos forestales no maderables en Iquitos-Perú. Folia Amazonica, 11 (1-2): 181-192.

Barcelos E., G. Second, F. Kahn, P. Amblard, P. Lebrun \& M. Seguin. 1999. Molecular markers applied to the analysis of genetic diversity and to the biogeography of Elaeis. Memoirs New York Botanical Garden, 83: 191-201.

Barriga R. 1994. Plantas útiles de la Amazonía Peruana: características, usos y posibilidades. CONCYTEC, Editorial Libertad, Lima.

Berlin B. 1970. A preliminary ethnobotanical survey of the Aguaruna region of the upper Marañón river valley, Amazonas, Peru, Washington D.C., Report for the Wenner-Gren Foundation for Anthropological Research.

Berlin B. 1977. Sumario de la primera expedición etnobotánica al río Alto Marañón, Dpto. de Amazonas. Amazonia Peruana - ecología, V.1 (2): 87-100.

Berlin B. 1992. Ethnobiological classification. Principles of categorization of plants and animals in traditional societies. Princeton University Press, New Jersey.

Berlin B. \& E.A. Berlin. 1979a. Etnobiología subsistencia y nutrición en una sociedad de la selva tropical: los Aguaruna, Centro de Investigación y Promoción Amazónica, Perú, pp. 13-49.

Berlin B. \& E.A. Berlin. 1979b. Aspectos de la etnobiología Aguaruna-Huambisa, segunda expedición etnobiológica al río Alto Marañón. Amazonas-Perú, Universidad de California, Berkeley.

Bernal R.G. 1992. Colombian palm products. In: M. Plotkin, Famolare, L. (eds), Sustainable harvest and marketing of rain forest products, Island Press/Conservation Internacional, Washington, pp. 158-172.

Blaack G. 1983. Procesamiento de los frutos de la palmera cucurita (Maximiliana maripa). In: Palmeras poco utilizadas de América tropical, FAO-CATIE, Turrialba, pp. 113-117.

Bodley J. H. 1978. Preliminary ethnobotany of the Peruvian Amazon. Reports of Investigations, ${ }^{\circ} 55$, Washington, $77 \mathrm{p}$.

Bodley J.H. \& F.C. Benson. 1979. Cultural ecology of Amazonian palms. Reports of Investigaciones $n^{\circ} 56$. Laboratory of Amazonian anthropology. Washington State University, Pullman.

Bodmer R.E., P.E. Puertas, J.E. García, D.R. Dias \& C. Reyes, 1999. Game animals, palms, and people of the flooded forests: management considerations for the Pacaya-Samiria national reserve, Peru. Advances in Economic Botany, 13: $217-231$.

Bohórquez J.A. 1976. Monografia sobre Mauritia flexuosa L.f. In: Simpósio internacional sobre plantas de interés económico de la flora amazónica. IICA, Informes, Cursos y Reuniones, 93: 223-248.

Boll T., J.-C. Svenning, J. Vormisto, S. Normans, C. Grández \& H. Balslev. 2005. Spatial distribution and environmental preferences of the piassaba palm Aphandra natalia (Arecaceae) along the Pastaza and Urituyacu rivers in Peru. Forest Ecology and Management, 18: 175-183.
Borchsenius F. \& M. Moraes. 2006. Diversidad y usos de palmeras andinas (Arecaceae), in: Moraes, M., B. Ollgaard, F. Borchsenius \& H. Balslev (eds.). Botánica Económica de Los Andes Centrales, Universidad Mayor de San Andrés, La Paz, pp. 412-433.

Borchsenius F., H. Borgtoft Pedersen \& H. Balslev. 1998. Manual of the palms of Ecuador. AAU Report 37, Aarhus University Press, Aarhus.

Borgtoft Pedersen H. 1992. Use and management of Aphandra natalia (Palmae) in Ecuador. Bulletin de l'Institut français d'Études andines, 21 (2): 741-753.

Borgtoft Pedersen F. \& H. Balslev. 1990. Ecuadorean palms for agroforestry. AAU Reports 23, Aarhus.

Brack A.E. 1999. Diccionario enciclopédico de plantas útiles del Perú. Centro Bartolomé de Las Casas, Cusco.

Bresani E. 1924. La palmera "aguaje" como productora de celulosa. Boletín de la Sociedad Geográfica de Lima. Tomo XLI: 209-210.

Byg A. \& H. Balslev. 2004. Factors affecting local knowledge of palms in Nangaritza Valley in South-Eastern Ecuador. Journal of Ethnobiology, 24(2): 255-278.

Campos J. \& A. Galán. 1989. Plantas empleadas por los habitantes del Dpto. de Amazonas. Boletín de Lima, 66: 57-60.

Campos Rivera A. 1988. Estudio de extracción por solventes y caracterización físico-química del aceite de suri (Rhynchophorus palmarum L.). Tesis Ing $^{\circ}$ Ind. Aliment., UNALM, Lima.

Castagne C.C. 1971. Análisis estructural de un aguajal. Ministerio de Agricultura, zona agraria IX, Tarapoto, informe, $9 \mathrm{p}$.

Castro A. 1993. Extractive exploitation of the açaí near Manaus, Amazônia. In: Pagesy, H., O.F. Linares, A. Hladik \& M. Hadley (eds). Food and Nutrition in the tropical forest: Biocultural interactions, Man and the Biosphere series, Vol. 15 Parthenon Publ Group, pp. 779-782.

Cavalcante P.B. 1974. Frutas comestíveis da Amazônia. Museo Paraense Emílio Goeldi, Publicações avulsas 27, tome II, Belém, Pará.

Cavero E. R. 1941. La Amazonía Peruana. Torres Aguirre. Lima.

Chang A.S. \& L.Z. Aymara. 1987. La flora útil en las comunidades nativas del Alto Mayo. Informe Proyecto especial Alto Mayo, Ministerio de Agricultura.

Clement C.R. 1992. Domesticated palms. Principes, 36: 70-78.

Clement C.R. 1988. Domestication of the pejibaye (Bactris gasipaes, Arecaeae): past and present. Advances in Economic Botany, 6: 155-174.

Clement C.R., Mora Urpi, J.E., 1988. Pejibaye palm (Bactris gasipaes, Arecaeae): multi-use potential for the lowland humid tropics. Economic Botany, 41: 302-331.

Couvreur T.L.P., W.J. Hahn, J.-J. de Granville, J.-L. Pham, B. Ludeña \& J.-C. Pintaud. 2007. Phylogenetic relationships of the cultivated Neotropical palm Bactris gasipaes (Arecaceae) with its wild relatives inferred from chloroplast and nuclear DNA polymorphisms. Systematic Botany, 32 (3): $519-530$

Dañobeytia F.J.R. 2002. Especies forestales utilizadas en la construcción de la vivienda tradicional Asháninka en el ámbito del río Perene (Junín, Perú). Tesis, UNALM, Lima.

De Los Heros G.M.J. \& J.B. Zárate. 1980/1981. Posibilidades papeleras de pulpa al sulfato de pecíolos de aguaje. Revista Forestal del Perú, 10: 83-90.

Del Castillo D., E. Otárola \& L. Freitas. 2006. Aguaje. La maravillosa palmera de la Amazonía. IIAP, Iquitos.

Descola P. 1986. La nature domestique, Symbolisme et praxis dans l'écologie des Achuar. Éditions de la Maison des Sciences de l'Homme, Paris.

Descola P. 1987. La selva culta. Travaux de l'IFEA, tome 30, IFEA, Quito. 
Descola P. 1993. Les lances du crépuscule. Relations Jivaros, HauteAmazonie, Plon, Paris.

Didir L.M.A. 1995. Salud para todos. Plantas medicinales y salud indígena en la cuenca del río Madre de Dios, Perú. Bartolomé de las Casas, Cusco.

Dugand A. 1972. Las palmeras y el hombre. Cespedesia, 1: 31-103.

Flores Paitan S. 1998. Agroforesteria Amazónica: Una alternativa a la agricultura migratoria. In: Kalliola, R. \& S. Flores Paitan (eds). Geoecologia y Desarrollo Amazónico. Estudio integrado en la zona de Iquitos, Perú. Turun Yliopiston Julkaisuja. Annales Universitatis Turkuensis. Turun Yliopisto. Turku. Finlandia, pp. 417-440.

Gari J., 2001. Biodiversity and indigenous agroecology in Amazonia: The indigenous people of Pastaza. Etnoecologica, 5 (7): 21-37.

Gómez D., L. Lebrun, N. Paymal \& A. Soldi. 1996. Palmas útiles en la Provincia de Pastaza, Amazonia ecuatoriana. Manual práctico. Serie Manuales de plantas útiles amazónicas, $\mathrm{n}^{\circ} 1$, Quito.

González Boscán V.C. 1987. Los morichales de los llanos orientales. Un enfoque ecológico. Ed. Corpoven, Caracas.

González Rivadeneyra M. 1971. Estudio sobre la densidad de población de aguaje (Mauritia sp.) en Tingo María. Revista Forestal del Peru, 5: 46-54.

Goulding M. \& N. Smith. 2007. Palmeras. Centinelas de la conservación de la naturaleza. ACCA, ACA, Lima.

Govaerts R. \& J. Dransfield. 2005. World checklist of palms. Royal Botanic Gardens, Kew, UK.

Grenand P., C. Moretti, H. Jacquemin \& M.-F. Prévost. 2004. Pharmacopées traditionnelles en Guyane. IRD Editions, Paris.

Gullart J.-M. 1968. Nomenclatura Jibaro-Aguaruna de palmeras en el distrito de Cenepa. Biota, 57: 230-251

Gutiérrez H. 1987. Descripción, ubicación, aprovechamiento e importancia de tres palmeras de la Amazonía Peruana. Tesis, UNALM, Lima.

Henderson A. 1995. The palms of the Amazon. Oxford University Press, New York.

Henderson A. \& F. Chávez. 1993. Desmoncus as a useful palm in the western Amazon. Príncipes, 34: 184-186.

Henderson A., G. Galeano \& R. Bernal. 1995. Field guide to the palms of the Americas. Princeton University Press, New Jersey.

Holm Jensen O. \& H. Balslev. 1995. Ethnobotany of the fiber palm Astrocaryum chambira (Areacaceae) in Amazonian Ecuador. Economic Botany, 49 (3): 309-319.

Hübschmann L.K., L.P. Kvist, C. Grandez \& H. Balslev. 2007. Uses of Vara Casha - a Neotropical Liana Palm, Desmoncus polyacanthos - in Iquitos, Peru. Palms, 51 (4): 167-176.

Johnson D.V. 1975. Some palm products of the Peruvian Amazon. Príncipes, 19: 78-79.

Johnson D.V. 1986. Economic botany of the palm family in latin America and the Caribbean. Final report WWF 3322. Economic botany and threatened species in the palm family. Part 1 and 2.

Johnson D.V. \& K. Mejía. 1998. The making of a dugout from the trunk of the palm Iriartea deltoidea. Príncipes, 42: 201-205, 208.

Jordán Ledesma M.E. 1986. Estudio del fruto de aguaje (Mauritia flexuosa) como fuente de materia grasa para consumo humano. Tesis Ing ${ }^{\circ}$ Ind. Aliment., UNAP, Iquitos.

Kahn F. 1988. Ecology of economically important palms in Peruvian Amazonia. Advances in Economic Botany, 6: 42-49.

Kahn F. 1990. Palmeras nativas y manejo de bosques pantanosos de la Amazonía Peruana. Biota, 95: 58-63.
Kahn F. 1991. Palms as key swamp forest resources in Amazonia. Forest Ecology and Management, 38: 133-142.

Kahn F. 1993. Amazonian palms: food resources for the management of forest ecosystems. In: Hladik, C.M., H. Pagesy, O.F. Linares, A. Hladik \& M. Hadley (eds). Food and Nutrition in the tropical forest: Biocultural interaction. Man and the Biosphere series, Vol. 15 Parthenon Publ Group, pp. 153-162.

Kahn F. 1997. The Palms of El Dorado. Orstom, The International Palm Society, Éditions Champflour, Marly-le-Roi.

Kahn F. \& J.-J. de Granville. 1991. Los nombres vernáculos más comunes de las palmeras en la Amazonía. Biota 97: 17-32.

Kahn F. \& J.-J. de Granville. 1992. Palms in forest ecosystems of Amazonia. Springer Verlag, Berlin.

Kahn F. \& K. Mejía. 1986. The American oil palm, Elaeis oleifera, in Peruvian Amazonia. Principes, 30: 180.

Kahn F. \& K. Mejía. 1987. Notes on the biology, ecology, and use of a small Amazonian palm: Lepidocaryum tessmannii. Principes, 31: 215-219.

Kahn F. \& K. Mejía. 1988. Las palmeras de importancia económica en la Amazonía peruana. Folia Amazonica, 1 (1): 99-112.

Kahn F. \& F. Moussa. 1994. Las palmeras del Perú - Colecciones, Patrones de distribución geográfica, ecología, estatutos de conservación, nombres vernáculos, utilizaciones. IFEA, Lima.

Kahn F. \& F. Moussa. 1995. Les migrations de palmiers provoquées par l'homme en Amazonie et à sa périphérie. Biogeographica, 71 (4): 161-177.

Kahn F., K. Mejía, F. Moussa \& D. Gómez. 1993. Mauritia flexuosa, la más acuática de las palmeras amazónicas. In: Kahn, F., B. León \& K. Young (eds). Las plantas vasculares en las aguas continentales del Perú, IFEA, Lima, pp. 287-308.

La Torre-Cuadros M. A. 1998. Etnobotánica de los recursos vegetales silvestres del caserío de Yanacancha, Distrito de Chumuch, Provincia de Celendín, Departamento de Cajamarca. Tesis UNALM, Lima.

Lévi-Strauss C. 1950. The use of wild plants in tropical South America. Handbook of South American Indians 6, Cooper Square publishers, New York, pp. 465-486.

Lleras E. 1985. Estrategias para la domesticación de frutales nativos de la Amazonía. Workshop sobre cultivos nativos - INIPA/ IICA, San Ramón, Chanchamayo, Perú. Documento de trabajo, $28 \mathrm{p}$.

Lleras E. \& L. Coradin. 1988. Native neotropical oil palms: state of the art and perspective for Latine America. Advances in Economic Botany, 6: 201-213.

Lognay G., E. Trebejos, E. Jordán, M. Marlier, M. Severin \& O. de Zárate. 1987. Investigaciones sobre el aceite de Mauritia flexuosa L.f. Grasas y Aceites, 38 (5): 303-307.

López C.R. 1968. Ensayos de germinación de Mauritia flexuosa L.f. UNAP, Iquitos, $2 \mathrm{p}$.

López Mori J.A. 1984. Estúdio de tratamientos pregerminativos y manejo de semillas de Mauritia flexuosa L.f. (aguaje), Iquitos, Peru. Tesis Ing ${ }^{\circ}$ forestal, UNAP, Iquitos.

López Parodi J. 1988. The use of palms and other native plants in non-conventional, low coast rural housing in the Peruvian Amazon. Advances in Economic Botany, 6: 119-129.

Mariath J.G.R., M.C.C. Lima \& L.M.P. Santos. 1989. Vitamine A activity of Buriti (Mauritia vinifera Mart.) and its effectiveness in the treatment and prevention of xerophtalmia. American Journal of Clinical Nutrition, 49(5): 849-853.

Martius C.F.P. von, 1844 (1847). Palmetum Orbignianum. In: A. d'Orbigny, Voyage dans l'Amérique méridionale, 7 (3): 1-140, Paris.

Mejía K. 1983. Palmeras y el selvícola amazónico. UNMSM, Museo de Historia Natural, Lima, 6p. 
Mejía K. 1988. Utilization of palms in eleven Mestizo villages of the Peruvian Amazon (Ucayali River, Department of Loreto). Advances in Economic Botany, 6: 130-136.

Mejía K. 1992. Las palmeras en los mercados de Iquitos. Bulletin de l'Institut français d'Études andines, 21 (2): 755-769.

Mejía K. \& E. Rengifo. 1995. Plantas medicinales de uso popular en la Amazonía Peruana. AECI-CAAAP, Lima

Millán B. 1998. Estudio etnobotánico y taxonómico de especies amazónicas del género Astrocaryum (Arecaceae). Loreto, Madre de Dios. Tesis Magíster en Botánica tropical, UNMSM, Lima.

Montufar R.G. 2007. Structure génétique, biochimique, morphologique et écologique de Oenocarpus bataua Mart. (Arecaceae) : perspectives pour la valorisation durable d'une ressource forestière néotropicale. Thèse Doctorat, Supagro, Montpellier.

Moore Jr. H.E., A.C. Salazar \& E.E. Smith. 1960. A reconnaissance survey of palms in eastern Peru. Agricultura division, United States operation mission to Peru, Internacional cooperation administration, Lima.

Moussa F. \& F. Kahn. 1997. Trois palmiers pour trois capitales amazoniennes. Bulletin de l'Institut français d'Études andines, 26 (1): 1-9.

Moussa F., E. Lleras, G. Couturier \& F. Kahn. 1998. Alguns aspectos metodológicos em botânica econômica: caso das palmeiras amazônicas. In: Fonseca V.S., I.S. Machline \& C.F.C. de Sá (eds). Etnobotânica, bases para a conservação. I workshop de Etnobotânica e Botânica Econômica. Nova Friburgo, SBB, Rio de Janeiro, pp. 9-49.

Noriega Monteiro V.R. 1980. Colección del fruto del aguaje y su transformación en polvo. Tesis Ing $^{\circ}$ forestal, UNALM, Lima.

Padoch C. 1988. Aguaje (Mauritia flexuosa L.f.) in the economy of Iquitos, Peru. Advances in Economic Botany, 6: 214-224.

Padoch C. \& W. de Jong. 1990. Santa Rosa: the impact of the forest products trade on an Amazonian place and population. Advances in Economic Botany, 8: 151-158.

Paniagua Zambrano N.Y. 2005. Factors determining the knowledge and use of palms in the western Amazon. Tesis University of Aarhus.

Paniagua Zambrano N.Y., A. Byg, J.-C. Svenning, M. Moraes, C. Grandez \& H. Balslev. 2007. Diversity of the palm uses in the western Amazon. Biodiversity and Conservation, 16: 2771-2787.

Paramonga 1965. Pulpa experimental del aguaje (Mauritia vinifera). Paramonga, $3 \mathrm{p}$.

Pennington T.D., C. Reynel \& A. Daza. 2004. Ilustrated guide to the trees of Peru. David Hunt, Sherborne, England.

Pérez D. 2002. Etnobotánica medicinal y biocidas para malaria en la Región Ucayali. Folia Amazonica, 13 (1-2): 87-108.

Pesce C. 1985. Oil palms and others oil seeds of the Amazon. Translated and cited by D. Johnson from the original, Oleaginosas da Amazônia 1941. Oficina Graficas da Revista da Veterinaria, Belém. Reference Publications, Algonac, Michigan.

Peters C.M. \& E.J. Hammond. 1990. Fruits from the flooded forests of Peruvian Amazonia. Yield estimates for natural populations of three promising species. Advances in Economic Botany, 8: 159-176.

Peters C.M., A.H. Gentry \& R. Mendelsohn. 1989a. Valuation of an Amazonian rainforest. Nature, 339: 655-656.

Peters C.M., M.J. Balick, F. Kahn \& A.B. Anderson. 1989b. Oligarchic forests of economic plants in Amazonia: utilization and conservation of an important tropical resource. Conservation Biology, 3: 341-349.
Phillips O.L.B., 1993. The potential for harvesting fruit in tropical rainforests: new data from Amazonian Peru. Biodiversity and Conservation 1: 18-38.

Piñedo-Vásquez M., D. Zarin \& P. Jipp, 1992. Economic returns from forest conversion in the Peruvian Amazon. Ecological Economics, 6: 163-173.

Piñedo-Vásquez M., D. Zarin, P. Jipp \& M. Chota-Inuma. 1990. Use of tree species in a communal forest reserve in northeast Peru. Conservation Biology, 4: 405-416.

Pintaud J.-C., G. Galeano, H. Balslev, R. Bernal, F. Borchsenius, et al. 2008. Las palmeras de América del Sur: diversidad, distribución e historia evolutiva. Rev. per. biol. 15(supl. 1): 7-29

Pinto G.P. 1951. O óleo de patauá. Bol. Tec. IAN, Belém, 23: 67-77.

Ramírez E.A. 1974. Algunos aspectos de la germinación de aguaje (Mauritia flexuosa L.f.) en diferentes estratos germinativos y tratamientos de semillas. Tesis $\mathrm{Ing}^{\circ}$ agrónomo, Universidad Agraria La Selva, Tingo Maria.

Ramírez N. \& Y. Brito, 1990. Reproductive biology of a tropical palm swamp community in the Venezuelan llanos. American Journal of Botany, 77 (10): 1260-1271.

Rengifo E. \& T. Cerruti. 1997. Plantas medicinales de la Amazonía peruana: estudio de su uso y cultivo. IIAP, Iquitos.

Roca F. 2004. Ethnobotanique Aguaruna-Huambisa, le cas des palmiers et des orchidées dans les systèmes de classification. Tesis Doctoral, EHESS-París.

Rocha E. 2004. Potencial ecológico para o manejo de frutos de açaizeiro (Euterpe precatoria Mart.) em áreas extrativistas no Acre, Brasil. Acta Amazonica 34(2): 237-250.

Rojas Ruiz R. 1985. Ensayos de germinación con semillas de 5 especies de palmeras aplicando 10 tratamientos pregerminativos y ensayos de cosecha con 7 métodos. Tesis Ingeniero forestal, UNAP, Iquitos.

Rojas Ruiz R. 1992. Aspectos morfométricos de la chambira (Astrocaryum chambira) en áreas libres y boscosas de Jenaro herrera - rio Ucayali. Folia Amazónica 4 (2): 45-51.

Ruiz Murrieta J. 1991. El aguaje, alimento del bosque amazónico. COTESU, Región Ucayali, Temas Forestales 8, 28 p.

Ruiz Murrieta J. 1993. Alimentos del bosque amazónico: una alternativa para la protección de los bosques tropicales. UNESCO/ORCYT, Montevideo.

Ruiz Murrieta J. \& J. Levistre Ruiz, 1993. Extractivism in the Amazonian forest in Peru: the "aguajales" case study. In: Hladik, C.M., H. Pagesy, O.F. Linares, A. Hladik $\&$ M. Hadley (eds). Food and nutrition in the tropical forest: biocultural interactions, UNESCO, Man and the Biosphere series Vol. 13, Parthenon Publ. Group., Paris, pp. 797-804,

Salazar A. 1967. El aguaje, (Mauritia vinifera) recurso forestal potencial. Revista Forestal del Perú, 1: 65-68.

Salazar A. \& J. Roessl. 1977. Estudio de la potencialidad industrial del aguaje. Proyecto ITINTEC 3102 UNA-IIA, Lima.

Salo J. \& J. Torres Vásquez. 1998. Potencialidades de uso del recurso biodiversidad en Loreto. In: Kalliola, R., Flores Paitan, S. (eds), Geoecología y Desarrollo Amazónico. Estudio integrado en la zona de Iquitos, Perú. Turun Yliopiston Julkaisuja. Annales Universitatis Turkuensis. Turun Yliopisto. Turku. Finlandia, pp. 493-512.

Schultes R.E. 1977. Promising structural fiber palms of the Colombian Amazon. Príncipes, 21: 72-82.

Schultes R.E. \& R.F. Raffauf. 1990. The healing forest. Medicinal and toxic plants of the northwest Amazonia. Dioscorides Press, Portland, Oregon.

Schwyzer A. 1978. Producción casera del aceite de ungurahui (Jessenia polycarpa). Boletín técnico, proyecto de asentamiento rural integral Jenaro Herrera, Iquitos, $11 \mathrm{p}$. 
Soukup J. 1962. Vocabulario de los nombres vulgares de la flora peruana. Biota, IV (31): 102-144, (32): 164-192, (33): 205-236, (34): 259-280.

Soukup J. 1963. Vocabulario de los nombres vulgares de la flora peruana. Biota, IV (35): 281-316.

Soukup J. 1964. Vocabulario de los nombres vulgares de la flora peruana. Biota, V (37): 6-41.

Stagegaard J., M. Sorensen \& L.P. Kvist. 2002. Estimation of the importance of plant resources extracted by inhabitants of the Peruvian Amazon floodplains. Perspectives in Plant Ecology. Evolution and Systematic, 5: 103-122.

Testua Vásquez L.A. \& J.C. Aspajo Dávila. 1987. Estúdio de la extracción de caroteno (pro-vitamina A) a partir del aguaje (Mauritia flexuosa L.f.). Tesis Ingeniero químico, UNAP, Iquitos.

Tournon J. 2002. La merma mágica. Vida e historia de los ShipiboConibo del Ucayali. CAAAP, Lima.

Tournon J., G. Serrano, R. Reategui \& J. Albán. 1986. Plantas y árboles medicinales de los Conibos del alto Ucayali: concepciones nativas y botánicas. Revista forestal del Perú, 13 (2): 107-136.

Trebejos E., G. Lognay, C.R. López \& E. Cerrón. 1991. Investigaciones sobre el aceite de ungurahui (Jessenia bataua). Conocimiento 2(1): 123-135.

Ugent D. \& C. M. Ochoa. 2006. La etnobotánica del Perú: desde la prehistoria al presente. Arecaceae, CONCYTEC, Lima, pp. 56-64.
Urrego Giraldo L.E. 1987. Estúdio preliminar de la fenelogía de la canangucha (Mauritia flexuosa L.f.). Colombia Amazonica, 2: 57-81.

Van Der Linden M. \& C.R. López. 1990. Utilización de palmeras amazónicas en el Nororiente peruano. Revista Forestal del Perú, 17 (1): 65-74.

Vásquez R. 1999. Sistemática de las plantas medicinales de uso frecuente en el área de Iquitos. Folia Amazonica, 4 (1): 61-76.

Vásquez R. \& A.H. Gentry. 1989. Use and misuse of forest-harvested fruits in the Iquitos área. Conservation Biology, 3: 1-12.

Vormisto J. 2002 (a). Palms as rainforest resources: how evenly are they distributed in Peruvian Amazonia? Biodiversity and Conservation, 11: 1025-1045.

Vormisto J. 2002 (b). Making and marketing chambira hammocks and bags in the village of Brillo Nuevo, northeastern Peru. Economic Botany, 56: 27-40.

Wallace A.R. 1853. Palm trees of the Amazon and their uses. John van Voorst, London.

Wheeler M.A. 1970. Siona use of chambira palm fiber. Economic Botany, 24: 180-181.

Yacovleff E. \& F.L. Herrera. 1935. El mundo vegetal de los antiguos peruanos. Revista del Museo Nacional, IV (1): 31-102.

Yákuam'Jintia \& Péas Kantáshichtik, 1991. Achuar matsátmau: mundo achuar. $\mathrm{N} .^{\circ} 1$, Centro amazónico de antropología y aplicación práctica, CAAAP, Lima. 191 p. 